\title{
Computation in a Reduced KII Network Based on Synchronization
}

\author{
Dongming $\mathrm{Xu}, *$ José C. Principe ${ }^{\dagger}$ \\ Computational NeuroEngineering Laboratory, Department of Electrical and \\ Computer Engineering, University of Florida, Gainesville, FL 32611, USA
}

\begin{abstract}
When implementing dynamical systems for information processing, a well-defined set of computational primitives should be constructed to represent the state space. Based on the specific collective behavior of Freeman's computational model of the olfactory cortex, we study the computation of reduced KII networks by using the synchronization of output channels. The design of coupling coefficients in the network for obtaining a desired output state response is presented. We demonstrate the computational power of reduced KII networks by two applications that address logic computation and associative memory, respectively. (C) 2006 Wiley Periodicals, Inc.
\end{abstract}

\section{INTRODUCTION}

Deterministic systems with complex dynamics have been studied in the past as information processors and feature extractors. Many approaches using oscillatory or chaotic networks ${ }^{1-6}$ have shown great computational potential in applications such as pattern recognition, associative memory, and image processing. However, the dynamical approach for information processing is much less developed than the statistical reasoning used in pattern recognition. Only recently did nonlinear dynamics start being used to describe computation, ${ }^{7}$ and there remains a long way to achieve a nonlinear dynamical theory of information processing. The challenge is particularly important in the case of Freeman's model. The computational model of the olfactory system proposed by Freeman describes brain function as a spatiotemporal lattice of groups of neurons (neural assemblies) with dense interconnectivity. ${ }^{1}$ It quantifies the function of one of the oldest sensory cortices, where there is an established causal relation between stimulus and response. It also presents the function as an association between stimulus and stored information, in line with the content addressable memory (CAM) framework studied in artificial neural networks. ${ }^{8}$ This distributed system is locally

Author to whom all correspondence should be addressed: e-mail: dmxu@cnel.ufl.edu.

†e-mail: principe@cnel.ufl.edu.

INTERNATIONAL JOURNAL OF INTELLIGENT SYSTEMS, VOL. 21, 919-935 (2006)

(C) 2006 Wiley Periodicals, Inc. Published online in Wiley InterScience

(www.interscience.wiley.com). • DOI 10.1002/int.20169 
stable but globally unstable, creating nonconvergent (eventually chaotic) dynamics. Nonconvergent dynamics are very different from the simple dynamical systems with point attractors studied by Hopfield, ${ }^{9}$ because they have positive Lyapunov exponents. ${ }^{10}$ Freeman utilizes the language of dynamics to model neural assemblies, which seems a natural solution based on the known spatiotemporal characteristics of brain function. ${ }^{11}$ The model has been applied to many information processing applications. ${ }^{12-15}$

To accomplish a desired task, a reasonable interpretation of state space should be the basis of every computational system. A well-defined set of computational primitives should be constructed to represent the state space. Different dynamics will provide different sets of primitives; therefore, theoretical analysis should be conducted to provide reasoning for why the given primitives are the best. This is certainly dependent on the system's fundamental structure. Among many examples of information processing using dynamical systems, a well-studied case is creating attractors (either convergent or nonconvergent) in state space. Properties such as convergency and bifurcation are used to construct the fundamental computational primitives. The case of dynamical systems with point attractors (as in the Hopfield network) generate local minima in the energy function, and their study as associative memories has produced understanding and information processing properties. The case of nonconvergent dynamical systems such as the Freeman model is much harder to quantify. Attractors in the network have been studied, and various methods are applied to quantify the global behavior of the networks for pattern recognition applications. However, the design of an optimal readout for the spatiotemporal dynamics has not been thoroughly investigated, which shows that fundamental understanding of the dynamical primitives is needed.

In this article, we propose to use synchronization in the output space of the Freeman model as the readout. In many other oscillatory networks, ${ }^{5,16-18}$ synchronization among groups of oscillators has been used in various applications such as image segmentation, audio processing, and pattern recognition. More interestingly, phase coding ${ }^{19}$ has also been studied for Freeman's model, which shows advantages over conventional pattern recognition techniques based on amplitude. In this article, more specifically, we will not only use synchronization to construct output space but also use it directly in designing the structure of the network based on the desired functionalities we want to achieve. In this complicated and highly interconnected network, in response to input stimulus, the dynamics collapse to a lower dimensional space, and simple binary decisions can categorize different groups of oscillators. Actually, global behaviors that are generated by interactions among lower level components can be very rich. There exists a wide variety of collective behaviors in both physical and biological systems including synchronization, partial synchronization, desynchronization, dephasing, phase trapping, bursting in coupled neural oscillators through diffusive coupling 20 and strongly coupled integrate-and-fire oscillators, ${ }^{21}$ chaos in two coupled van de Pol oscillators, ${ }^{22}$ synchronization and traveling waves in an array of integrate-and-fire neurons with weak coupling ${ }^{23}$ time-delay-induced amplitude death in coupled oscillators, ${ }^{24-26}$ and synchronization of brain activity. ${ }^{27-30}$ These provide for a huge quantity of possible quantitative measures to refine the state space of our system to construct 
different representations of embedded signals. If they can all be utilized, both computational ability and efficiency will be greatly increased.

Based on our previous work on analyzing dynamical behaviors of basic components in the Freeman model, ${ }^{31,32}$ we will show how to design critical parameters in the network to obtain desired output responses, especially synchronized and desynchronized behaviors. Two applications will be presented. The first application provides a different view of computation in Freeman's model. ${ }^{33}$ With fixed coupling coefficients, a combination of external inputs will lead the coupled oscillators in a reduced KII network to two different states: synchronization and desynchronization. It turns out that with only two oscillators, by determining the proper values of the coupling coefficients, we can build functional blocks to implement two-input Boolean logic gates including AND, OR, XOR, NAND, NOR, XNOR, and NOT. In the second application, an associative memory is constructed by using synchronization among output channels in a reduced KII network as the readout. The network turns out to be more robust than those designed by conventional methods.

The rest of the article is organized as follows. In Section 2, we introduce the mathematical model of olfactory cortex. Section 3 presents the methodology of synchronization analysis and analytical solution of coupling strength to achieve synchronization in coupled reduced KII (RKII) sets. Section 4 presents computations in RKII network. Section 5 concludes the article.

\section{COMPUTATIONAL MODEL OF THE OLFACTORY CORTEX}

Freeman's model is a locally stable but globally chaotic system with very high dimensionality. The complexity of the whole system is expressed as a hierarchical embedding of simpler structures. Four different levels are included as K0, $\mathrm{KI}, \mathrm{KII}$, and KIII, where K stands for Katchalsky. ${ }^{1}$ It describes the nervous system as a spatiotemporal lattice of nonlinear processing elements (PEs) of the form

$$
\begin{aligned}
\frac{1}{a \cdot b} \cdot & {\left[\frac{d^{2}}{d t^{2}} x_{i}(t)+(a+b) \cdot \frac{d}{d t} x_{i}(t)+(a \cdot b) \cdot x_{i}(t)\right] } \\
& =\sum_{j \neq i}^{N}\left[W_{i j} \cdot Q\left(x_{j}(t), q_{j}\right)+W_{i j}^{\prime} \cdot f_{j}\left(Q\left(x_{j}(t), q_{j}\right), t\right)\right]+P_{i}(t), \quad i=1, \ldots, N
\end{aligned}
$$

Each PE in Equation 1 is a K0 set. Every K0 set has three major blocks: a summing node, second order dynamics, and an asymmetric nonlinear block. The nonlinear function $Q(x)$ models the spatiotemporal integration of spikes into mesoscopic waves measured in the cortex. ${ }^{1} 1 / a$ and $1 / b$ are time constants of the secondorder dynamics, and $Q(x)$ is defined as

$$
Q(x)= \begin{cases}Q_{m} \cdot\left(1-e^{-\left(e^{x}-1\right) / Q_{m}}\right) & x>x_{0} \\ -1 & \text { else }\end{cases}
$$




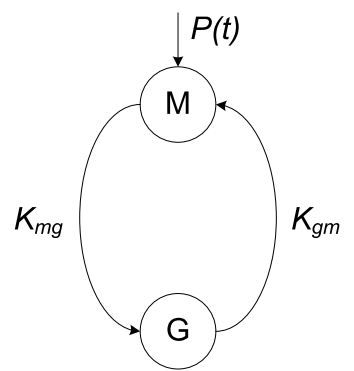

(a)

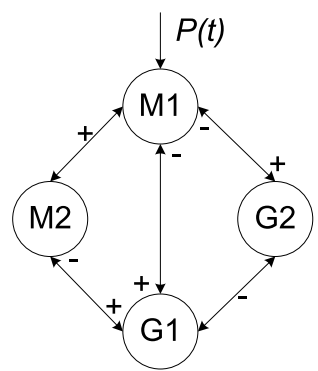

(b)

Figure 1. An RKII set shown in (b) consists of one mitral PE and one granule PE that are coupled through $K_{m g}(>0)$ and $K_{g m}(<0)$. It is a simplified version of a KII set shown in (a).

where $x_{0}=\ln \left(1-Q_{m} \cdot \ln \left(1+1 / Q_{m}\right)\right)$. Two kinds of K0 sets are distinguished by their signs of the nonlinear gain. The second level KI has interconnected K0 sets with only positive (excitatory) or negative (inhibitory) connections. A KII set is composed of four K0 sets. Two of them are excitatory whereas the other two are inhibitory. Basically, the KII set is an oscillator whose behavior is controlled by an external input. A KII network is a network of coupled oscillators (KII), in which different KII sets are interconnected through excitatory cells and inhibitory cells. A KIII network that consists of all the previous levels describes the behavior of the olfactory cortex. ${ }^{1}$

A single RKII set (Figure 1a) consists of two equations from Equation 1 that are internally coupled through excitatory connection $K_{m g}(>0)$ and inhibitory connection $K_{g m}(<0)$. It is a simplified version of a KII set (Figure 1b) but provides for the same functionality in a KII network. ${ }^{1,15}$ By changing variables in Equation 1 , this system is formally defined by the following set of ODEs:

$$
\left\{\begin{aligned}
\dot{m}(t) & =m_{v}(t) \\
\dot{m}_{v}(t) & =-a b \cdot m(t)-(a+b) \cdot m_{v}(t)+a b \cdot\left(K_{g m} Q(g(t))+P\right) \\
\dot{g}(t) & =g_{v}(t) \\
\dot{g}_{v}(t) & =-a b \cdot g(t)-(a+b) \cdot g_{v}(t)+a b \cdot K_{m g} Q(m(t))
\end{aligned}\right.
$$

where $m(t)$ denotes the mitral PE and $g(t)$ denotes the granule PE.

As discussed in Ref. 31, RKII set is an input controlled oscillator. Given external input $P$, a bifurcation boundary is defined by

$$
\left|K_{m g} \cdot K_{g m}\right|=\frac{1}{Q^{\prime}\left(m^{*}\right) \cdot Q^{\prime}\left(g^{*}\right)} \cdot \frac{(a+b)^{2}}{a \cdot b}
$$

where $Q^{\prime}(x)$ is the derivative of $Q(x)$ and $\left(m^{*}, g^{*}\right)$ is the unique equilibrium ${ }^{31}$ of Equation 3. Particularly, when $P=0$, 


$$
\left|K_{m g} \cdot K_{g m}\right|=\frac{(a+b)^{2}}{a \cdot b}
$$

In this case, when $\left|K_{m g} \cdot K_{g m}\right|<(a+b)^{2} / a \cdot b$, Equation 3 is stable at the origin whereas it goes to a limit cycle when $\left|K_{m g} \cdot K_{g m}\right|>(a+b)^{2} / a \cdot b$.

A KIII network, which is a highly interconnected network of these basic elements (input controlled oscillators), is widely used in many applications related to pattern recognition. ${ }^{12-15}$ Conventional approaches train the network (train the coupling strength among mitral cells in the olfactory bulb [OB] layer) with adaptive methods such as Hebbian learning. However, training could be further simplified by assigning binary connections. That is, KII channels in the OB layer that are excited by the same pattern are strongly connected whereas those channels receiving zero input are weakly connected to all others. This approach is very efficient but may lack accuracy and demand great effort in trial-and-error experiments. Adaptive methods train the network automatically but may suffer convergence problem. In Ref. 32, an analytical solution for coupling strength to control the collective behaviors of RKIIs is presented. Based on the result, it is much easier to set the values of interconnection among excitatory (inhibitory) PEs from different RKII sets.

\section{SYNCHRONIZATION OF RKII SETS}

Synchronization of two dynamical systems have different definitions based on different purpose and applications. Generalized synchronization (GS) between a driving system and a response system is discussed in Ref. 34. In this article, we consider the case that follows the definition of identical synchronization (IS), in which the transformation from driving trajectory to response trajectory is an identity function. Different approaches to synchronization analysis of coupled chaotic systems have been discussed by many researchers. In Ref. 32, we studied the case of identical synchronization between two coupled identical RKII sets. Therefore, the two RKIIs have exactly the same parameters and receive identical inputs (Figure 2a). The analytical result to determine the coupling strengths that guarantee the synchronization between two linearly coupled RKII sets is given here. Although linearly coupled systems still serve our purpose well to construct computational systems using neural oscillators, it is still a much simplified model of the original system. Later we will also discuss the effect of nonlinear coupling based on the results on linear coupling. The coupled system can be described as

$$
\left\{\begin{array}{l}
\dot{m}^{(1)}=m_{v}^{(1)} \\
\dot{m}_{v}^{(1)}=-a b \cdot m^{(1)}-(a+b) \cdot m_{v}^{(1)}+a b \cdot\left(K_{g m} \cdot Q\left(g^{(1)}\right)+P+K_{m m} \cdot m^{(2)}\right) \\
\dot{g}^{(1)}=g_{v}^{(1)} \\
\dot{g}_{v}^{(1)}=-a b \cdot g^{(1)}-(a+b) \cdot g_{v}^{(1)}+a b \cdot\left(K_{m g} \cdot Q\left(m^{(1)}\right)+K_{g g} \cdot g^{(2)}\right)
\end{array}\right.
$$

International Journal of Intelligent Systems $\quad$ DOI 10.1002/int 


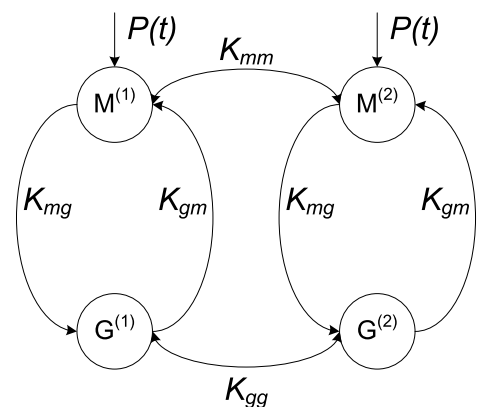

(a)

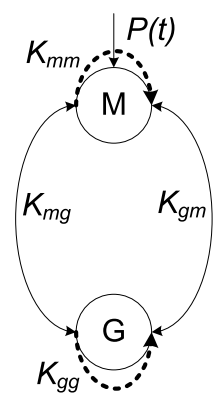

(b)

Figure 2. (a) Linearly coupled RKII sets. (b) Under synchronization, coupled RKII sets could also be described as an individual RKII set with auto-feedback.

where the output states from system 2 are coupled through $K_{m m}>0$ and $K_{g g}<0$. Denote the state vector as $\mathbf{X}\left(=\left[m, m_{v}, g, g_{v}\right]^{T}\right)$. We have

$$
\begin{aligned}
& \mathbf{X}^{(1)}=\mathbf{F}\left(\mathbf{X}^{(1)}\right)+\mathbf{E}\left(\mathbf{X}^{(2)}-\mathbf{X}^{(1)}\right) \\
& \mathbf{X}^{(2)}=\mathbf{F}\left(\mathbf{X}^{(2)}\right)+\mathbf{E}\left(\mathbf{X}^{(1)}-\mathbf{X}^{(2)}\right)
\end{aligned}
$$

where $\mathbf{X}^{(1)}, \mathbf{X}^{(2)}$, and $\mathbf{F} \in \mathbb{R}^{N}$. $\mathbf{E}_{N \times N}$ is the coupling matrix.

If we define the deviation of one system from another as $\mathbf{W} \equiv \mathbf{X}^{(1)}-\mathbf{X}^{(2)}$, the goal would be choose coupling strength so that $\mathbf{W}=\mathbf{0}$ is stable. Following Ref. 35, if we require both $K_{m m}$ and $\left|K_{g g}\right|$ to be limited in the unit square, then synchronization between two RKII sets can be achieved when the coupling coefficients satisfy $^{32}$

$$
\left(K_{m m}+\left|K_{g g}\right|\right)^{2}>4 K_{B}-4 K_{A 2}-2 K_{A 2} \cdot\left(K_{m m}-\left|K_{g g}\right|\right)
$$

and

$$
\left(K_{m m}+\left|K_{g g}\right|\right)^{2}<4 K_{B}^{*}+4 K_{A 2}+2 K_{A 2} \cdot\left(K_{m m}-\left|K_{g g}\right|\right)
$$

where

$$
\begin{aligned}
K_{A 1} & =\frac{(a-b)^{2}}{a b} \\
K_{A 2} & =\frac{(a+b)^{2}}{a b} \\
K_{B} & =\left|K_{m g} \cdot K_{g m} \cdot\left\langle Q^{\prime}\left(m^{(1)}\right)\right\rangle \cdot\left\langle Q^{\prime}\left(g^{(1)}\right)\right\rangle\right|
\end{aligned}
$$

and

$$
K_{B}^{*}=\left|K_{m g} \cdot K_{g m} \cdot Q^{\prime}\left(m^{*}\right) \cdot Q^{\prime}\left(g^{*}\right)\right|
$$


$\left\langle Q^{\prime}(x)\right\rangle$ is the measure of the derivative of $Q^{\prime}(x)$. The outputs are simple limit cycles, so we will use time average to evaluate them.

Equation 8 is the sufficient condition that guarantees synchronization of two coupled RKII sets. Equation 9 is a bifurcation boundary that is only valid when synchronization is achieved. It is to guarantee the appropriate behaviors after the coupled sets are synchronized. In addition, the bifurcation boundary is more accurate because it uses the exact values of equilibrium instead of estimations of limit cycles used in conditions on synchronization. The two boundaries are actually two parabolas (Figure 3a) that divide the parameter space into three possible regions, where two coupled RKII sets behave differently as synchronized oscillation, desynchronized oscillation, and fixed point. Figure $3 \mathrm{~b}$ gives an example of a detailed view of the actual boundaries within the unit square. The section of the bifurcation boundary that is below the synchronization boundary is removed. There are three other parameters $\left(K_{m g}, K_{g m}\right.$, and $\left.P\right)$ that can change the position and shape of the boundaries. Most of the time, these three parameters are predetermined, so we can calculate the boundaries accordingly. In the case where their values need to be changed, it is still easy to know how the boundaries move. In fact, only $K_{B}$ will be affected by the three parameters. Increasing or decreasing $K_{B}$ will shift the parabolas along the position vector $(1,-1)$ (Figure $3 \mathrm{a})$. Under identical synchronization, the two coupled RKII sets can be taken as a single oscillator that has autofeedback (Figure 2b). In an RKII network, when synchronization happens within groups of RKIIs, globally, the dimensionality of output space reduces to form new attractors while locally, within each group, we still see simpler dynamics that mimic those of single RKIIs. We will use this property later to build associative memories in RKII networks.

It is expected that in the case of nonlinearly coupled RKIIs as described in the original Freeman model, system behavior becomes very complicated. The above method does not directly produce accurate results for all the possible solutions on coupling strengths. Actually, from another point of view, we can regard nonlinear coupling as time-varying linear coupling. That is, at time $t, K_{m m} Q(m(t))$ (or $\left.K_{g g} Q(g(t))\right)$ can be linearized as $K_{m m} Q^{\prime}(m(t)) m(t)$ (or $\left.K_{g g} Q^{\prime}(g(t)) g(t)\right)$. The coefficients $K_{m m} Q^{\prime}(m(t))$ and $K_{g g} Q^{\prime}(g(t))$ are explicitly dependent on the system's trajectory. However, the lower bound of the fixed-point region as well as the bifurcation region in Figure $3 \mathrm{~b}$ are both determined using the fixed point at $(0,0)$ instead of limit cycle trajectories. Therefore, in these two cases, nonlinear coupling defaults to the linear case. Actually, simulations show that both nonlinear and linear coupling share exactly the same parameter space that includes the fixed-point region and the two oscillatory states right above and below the fixed-point region (Figure $3 b$ ). Coupling strength obtained for the linear case can be directly applied to nonlinear couplings in these regions. In practical applications, what matters is to visit all the interesting dynamics, for instance to train the readout. This can be achieved using only part of the parameter space. However, if we want to use a broader range of parameter values, either much thorough investigation or scanning of parameter space needs to be performed. In the following section, we present two applications that utilize the synchronization criterion with nonlinearly and linearly coupled RKII sets to serve as a readout for the RKII network. 

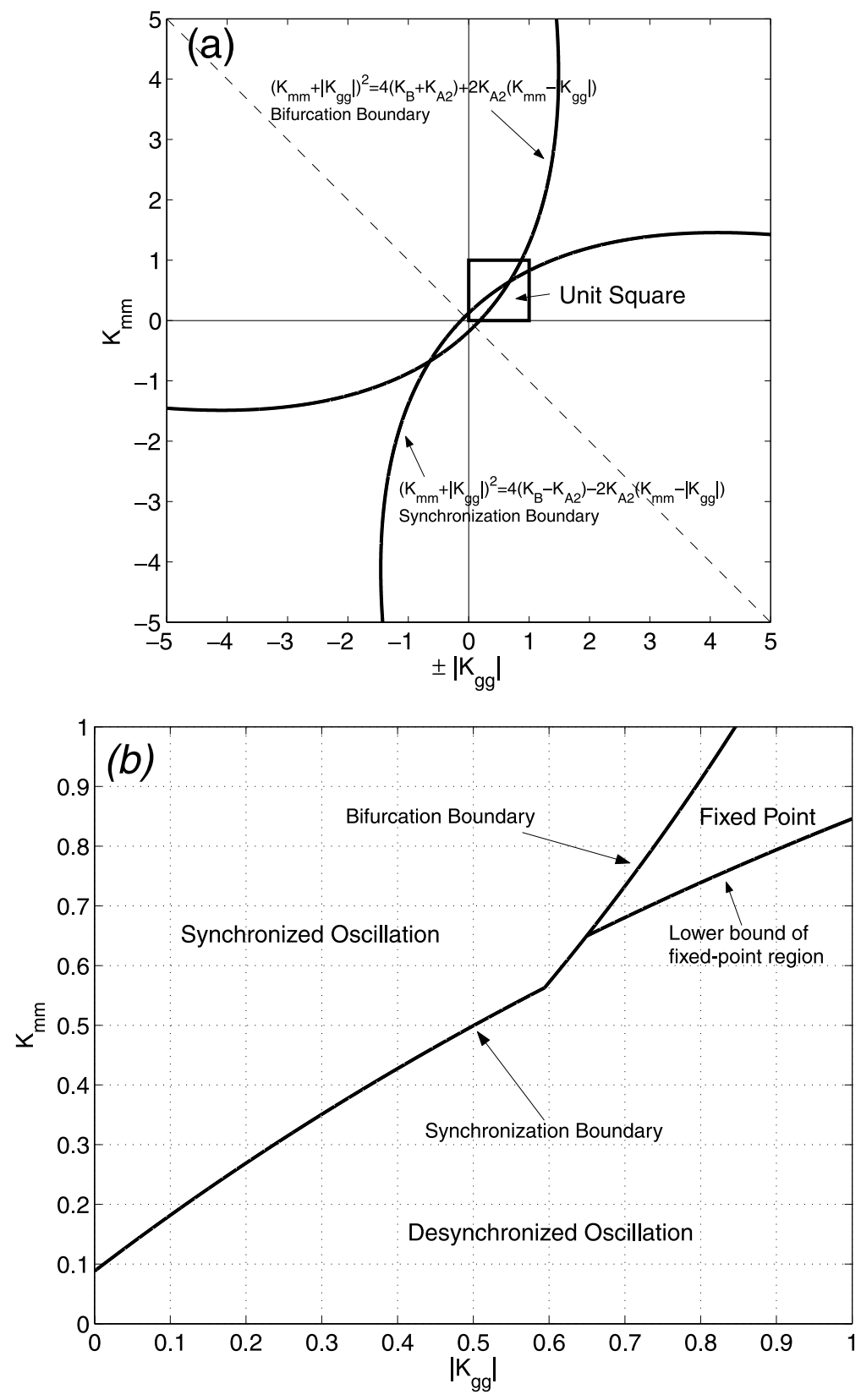

Figure 3. Parameter space defined by $K_{m m}$ and $K_{g g}$. (a) Equations 8 and 9 describe two parabolas. Normally, the system is defined within unit square. Changing $K B$ will move the two parabolas along the position vector $(1,-1)$. (b) The plot shows combined results of synchronization and bifurcation boundaries when input $P=0, K_{m g}=1$, and $K_{g m}=-6 .\left|K_{g g}\right|$ and $K_{m m}$ are limited by 1 . Three regions are determined where the coupled system shows synchronized oscillation, desynchronized oscillation, or fixed point solutions. The lower bound of the fixed-point region is estimated by Equation 9 using $K_{B}^{*}$. 


\section{COMPUTATION IN RKII NETWORK BASED ON SYNCHRONIZATION}

\subsection{Logic Computation}

From the previous section, we know that there are two basic behaviors for two coupled RKII sets. Based on the analysis, if we denote synchronization as state 1 and desynchronization as state 0 , the coupled system is a logic function when defined as

$$
f(\mathbf{I})=S\left(m^{(1)}, m^{(2)}, \theta\right)
$$

where $\mathbf{I}=\{0,1\} \times\{0,1\}$ is the input space and $S=\{0,1\}$ is any function that determines whether or not the outputs of the two systems $m^{(1)}$ and $m^{(2)}$ are synchronized. $\theta$ is the threshold used in $S$. Note that $\mathbf{I}$ is not limited to binary values of 0 and 1 . Basically, any two values that represent higher and lower level inputs can form the input space. Function $S$ is just a symbolic system where one can assign either 0 or 1 to any of the two states. This means that, if $f$ can implement one set of the basic logic computations (e.g., AND, OR, XOR), it should also be able to realize their complements with a different assignment of states in the actual measure of synchronization. The NOT function can also be implemented with many options. One example is to short the inputs of NAND gate or fix one of its inputs to 1. A two-input logic gate has a total of 16 Boolean functions. In this article, we only consider the case that the coupled oscillators have a symmetric structure, so it cannot distinguish between the inputs $(0,1)$ and $(1,0)$. However, this could be solved by using weighted inputs. The weights then become the parameters that need to be designed in addition to $K_{m m}$ and $K_{g g}$. By considering only the symmetric structure, without the combination of different gates, the coupled oscillators have the ability to implement 8 out of the 16 logic functions that include all the Boolean primitives. They cover most of the universal sets (e.g., $\{$ AND, OR, NOT $\}$ and $\{$ NAND $\}$ ) that can express any logic functions.

Synchronization can be measured using either correlations between the outputs of the two sets or direct detection of the phase difference. By these means, with a proper threshold, detection is not limited to perfect synchronization in the case when the inputs to the two oscillators are not identical. Equation $11 \mathrm{calcu}-$ lates the correlations between the time averagings of two state variables:

$$
R\left(m_{1}, m_{2}\right)=\frac{\left\langle m_{1} \cdot m_{2}\right\rangle-\left\langle m_{1}\right\rangle\left\langle m_{2}\right\rangle}{\sqrt{\left(\left\langle m_{1}^{2}\right\rangle-\left\langle m_{1}\right\rangle^{2}\right)\left(\left\langle m_{2}^{2}\right\rangle-\left\langle m_{2}\right\rangle^{2}\right)}}
$$

Notice that when the two sets are synchronized, the phase plot is a straight line. When they are desynchronized, there may be either a limit cycle or quasiperiodic motions in the phase plane. So another way is to plot the bifurcation diagram, so that multiple periods could be detected in the case of desynchronization.

In simulations, we fix $K_{m g}=\left|K_{g m}\right|=3$ and use $\{0,1\}$ as the input space. The two RKII sets are nonlinearly coupled. Different values of $K_{m m}$ and $K_{g g}$ are searched to see if all the six basic logic gates could be realized. We relax the limitations on 
Table I. Configurations for different logic functions.

\begin{tabular}{llllll}
\hline Function & Sync. & $K_{m m}$ & $K_{g g}$ & $K_{m g}$ & $K_{g m}$ \\
\hline AND (NAND) & $1(0)$ & 1.75 & -2.0 & 3 & -3 \\
OR (NOR) & $1(0)$ & 1.2 & -0.4 & 3 & -3 \\
XNOR (XOR) & $1(0)$ & 1.5 & -0.4 & 3 & -3 \\
\hline
\end{tabular}

the two parameters and allow them to have absolute values that are greater than one. Given the simple structure of just two coupled oscillators, the results are very promising. Using a synchronization criterion that assigns state 1 to synchronization and state 0 to desynchronization, the coupled RKII sets can implement AND, NOR, and XNOR functions. That is, by assigning 1 to desynchronization and 0 to synchronization, we have the complementary functions, that is, all the six logic functions. Figure $4 \mathrm{a}$, b shows the implemented XNOR function in the time domain and phase plane, respectively. Transient behaviors are discarded to guarantee a steady state of the outputs. The outputs of the nonlinear function, which have saturations at -1 and 5 , are plotted. In the case when inputs are [1 1$]$ and [0 0], the two reduced KII sets are perfectly synchronized. When inputs are $\left[\begin{array}{ll}1 & 0\end{array}\right]$ and $\left[\begin{array}{ll}0 & 1\end{array}\right]$, the two oscillators are desynchronized and even present chaotic behaviors. Note that, in this application, zero inputs to RKII sets still induce limit cycles. Actually, when both channels are synchronized, there are no chaotic behaviors locally or globally, no matter what the inputs are. This is different from the conventional Freeman model, where chaotic behaviors are always present. This is because here in the logic computation system, the output space is specifically designed to be either synchronized or desynchronized to construct binary functions. And as discussed previously, when two channels are synchronized, we expect the system to behave as a single RKII set that only has the limit cycle attractor. Table I gives the parameter values to build the logic gates. In the second column of the table, the label ( 0 or 1 ) defined for synchronized behaviors in $S$ is indicated.

One important question is how to extend this result to a network of oscillators. There are basically two structures. The first one is to implement the logic gates just as in digital design. That is, outputs from one set of coupled oscillators will be the input of the next one. And combinations of different gates will implement more complicated logic functions. The other way is to fully connect $N$ RKII sets. In this network, if we consider the synchronization of every pair out of the $N$ oscillators, the size of the binary output space could be as large as $2^{N}$ (although in practice, it may not be possible to design a network that can access all the possible outputs). This indicates the great potential of this network in terms of capacity.

\subsection{Associative Memory Using an RKII Network}

We consider the case where input patterns are static and only have binary values (positive and zero). The channels (RKIIs) that receive positive (zero) stimulus will be called active (inactive) channels. Ideally, oscillatory states of $m_{1} \mathrm{~s}$ that 
(a)
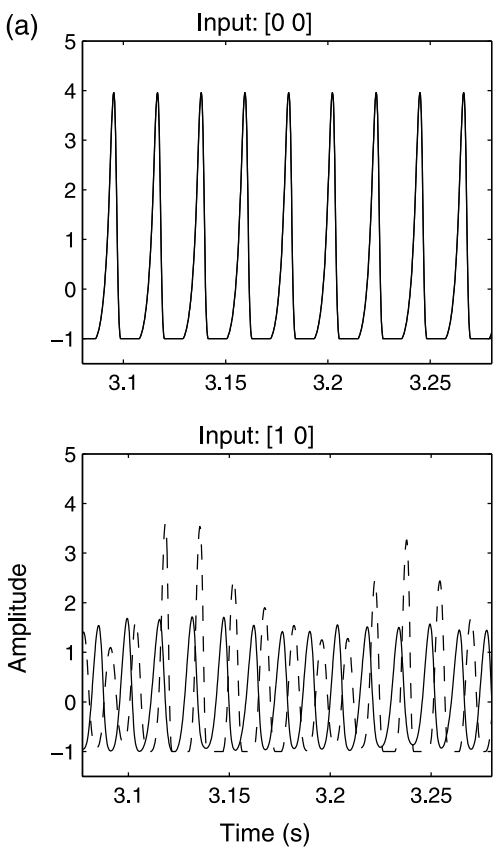

(b)
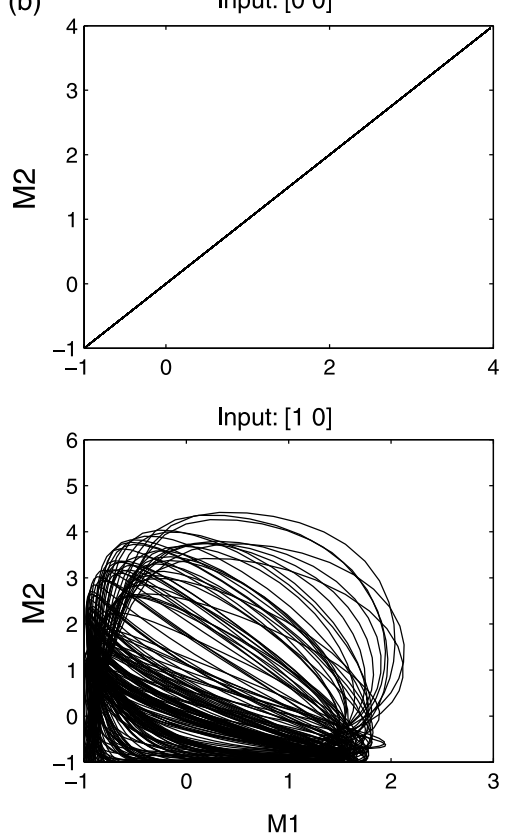
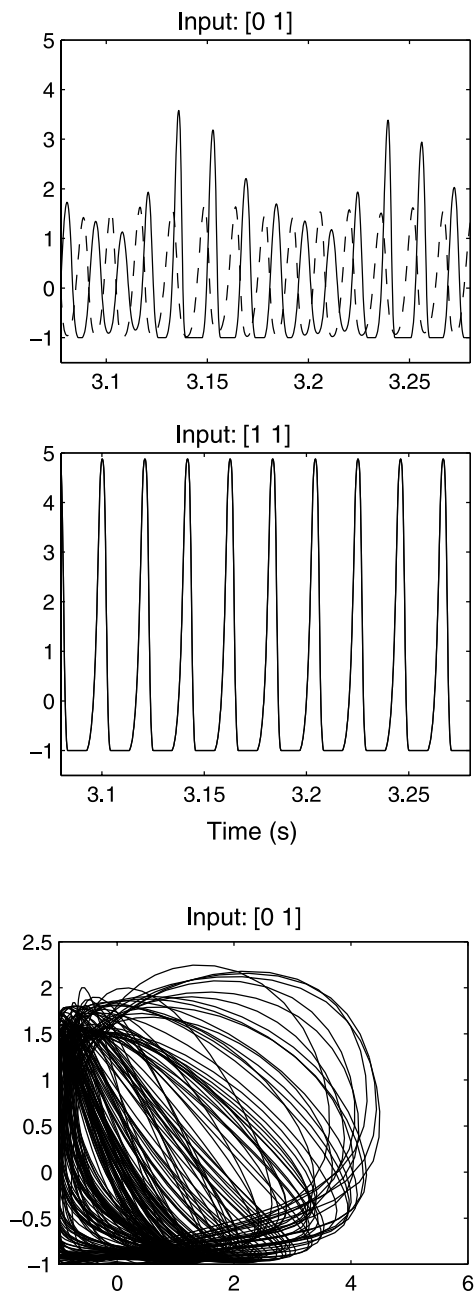

Input: [1 1]

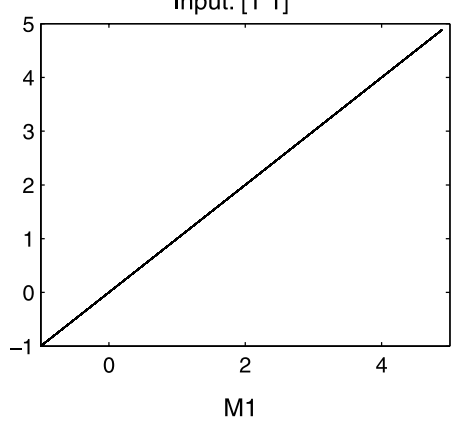

Figure 4. (a) Time domain response when implementing coupled RKII sets as an XNOR gate. State outputs are shaped by the nonlinear function. The solid line shows $Q\left(m^{(1)}\right)$ and the dashed line shows $Q\left(m^{(2)}\right)$. (b) Phase plot in the state space of $M_{1}$ and $M_{2}$ when implementing coupled RKII sets as an XNOR gate. 
are receiving the same inputs should be grouped together. That is, we expect zero phase lag among active channels. Under a strong constraint, we should expect only two kinds of different oscillations that are corresponding to high and low input values, respectively. However, in real applications, noise in the background or pattern distortions would make identical synchronization unrealistic. Here, we will still require active channels to be synchronized in the sense that phase differences between active channels only deviate from one another in a small neighborhood around zero. Behaviors of inactive channels and those caused by errors in the input space will be relaxed. Therefore, the only criterion for recognizing a pattern is to detect the active group that has strong synchronization. All other output states that are not strongly correlated to this group will be classified as null-state.

Many adaptive methods have been investigated for training the interconnection weights $\left(K_{m m}\right.$ and $\left.K_{g g}\right)$ in Freeman's model. Most of them are based on Hebbian learning ${ }^{12-14}$ that could also be simplified to assign binary values, strong or weak, to coupling coefficients among active or inactive channels. From Section 3, we know exactly how to control the behaviors of coupled RKII sets by different values of connections. Thus, coupling strength will be determined targeting specific desired behaviors. We consider four scenarios in the input space that specify the values of couplings between different channels.

To construct such a network as associative memory, based on excitation coming from input patterns, the following cases are utilized to determine coupling strength among different channels:

(1) Active channels. If two channels are to be excited by at least one of the stored patterns, when applying positive stimulus, we expect that the outputs from both channels are synchronized. At the same time, when receiving incomplete patterns, they should also be synchronized.

(2) Inactive channels. If two channels are never excited by any of the stored patterns, output states should be synchronized with weaker oscillations to be distinguished from the active channels. At the same time, if one or both of them are excited, they should be able to desynchronize from one another so that all of them would not be considered as active channels.

(3) Active and inactive channels. Without overlap between different channels, two channels that belong to active and inactive channels, respectively, within the same pattern should be always desynchronized.

(4) Overlapped channels. If two patterns share the same channel, connections between this mutual channel and other nonoverlapped channels will conflict for different patterns. That is, for one pattern, two channels are both active whereas it becomes Case 3 for another. In such situations, intuitively, we will reduce the inhibitory connections between this mutual channel and other possible active channels. Therefore, the activation of the mutual channel will be heavily dependent on the activations of other active channels in the incoming pattern but not affected much by active channels from another pattern that is not currently presented (inactive for the incoming pattern).

Based on the above conceptual design, we use the following as steps to set coupling strength for an RKII network. The network is initialized assuming Case 2. For every pattern, coupling strength between two active channels is set according to Case 1. Couplings between active and inactive channels are set according to Case 3 except for overlapped channels, which will be set according to Case 4 . 
The four cases are similar to the process of Hebbian learning. ${ }^{36,37}$ However, although the couplings are designed according to the properties of input-output correlations in both cases, the crucial difference lies in the structure of the readouts. Outer-product rule or adaptive algorithms such as Hebbian learning result in similarities among mutual channels in terms of energy. However, they cannot realize our goal to guarantee a solution that specifies the correlations such as phase information among output channels. Moreover, the values of couplings are calculated directly in our case instead of through learning, where issues such as convergence rate and stability may be a concern.

A 64-channel RKII network is implemented as an associative memory that is trained to memorize and recall patterns of digits " 1 " and " 0 " in the size of $8 \times 8$. To differentiate different groups of synchronized channels and their levels of activities, we use the following quantitative measure. First, cross correlations $R_{i, j} \mathrm{~s}$ between the $i$ th and $j$ th channels are calculated. Higher correlated channels are grouped together. Denote different groups as $G_{i}, i<N$, where $N$ is the total number of channels. The evaluation value for each group is simply described by the total amount of input received by all channels in the group $\left(\mathbf{O}_{i}=\sum_{k \in G_{i}} A_{k}\right.$, where $A_{k}$ is the input value received by the $k$ th channel). The same evaluation value is then assigned to all channels in the same group. Note that the network is designed such that only stored pattern will be recalled as synchronized output channels. Noise comes with higher input amplitude instead of zero but all output channels related to noise or false input will mostly not be synchronized and not be grouped together. Of course, we assume that noise or false input has an amplitude smaller than or at most comparable with ideal input values. For most cases, this is a simple but very effective criterion.

In the presence of clean patterns, the readout successfully recalls the patterns stored in the network (Figure 5a-d). In time domain, we see that all channels that receive positive stimuli are synchronized and have much higher amplitude. In this case, a simple energy readout would also work perfectly. However, when input distortion (incompleteness and noise) is presented, energy- or distance-based readout may fail, for example (Figure 5e,f), if input pattern " 1 " is colored by a positive stimulus to the channel that belongs to pattern " 0 ." In most cases, output level from this channel will be high enough to interfere with the desired active channels. The energy- and distance-based method in this case will have difficulty distinguishing false activities (noise) whereas the synchrony readout still extracts the phase information between channels efficiently. However, instantaneous detection of phase distributions may also fail because the frequency of oscillations would also be affected by external inputs. From experiments, oscillation frequency of the false channel is close to but not exactly the same as that of the true active channels, and the phase difference between the two is time varying. That is, if timing is not properly selected, instantaneous phase information will generate wrong information. However, in this case, long-term correlations between two channels still work. The window length used to track correlations depends on specific problems. Nevertheless, extra memory that stores a previous estimation of average values of output state and state powers will enable online update of cross correlations and save computation time needed for long time series. 

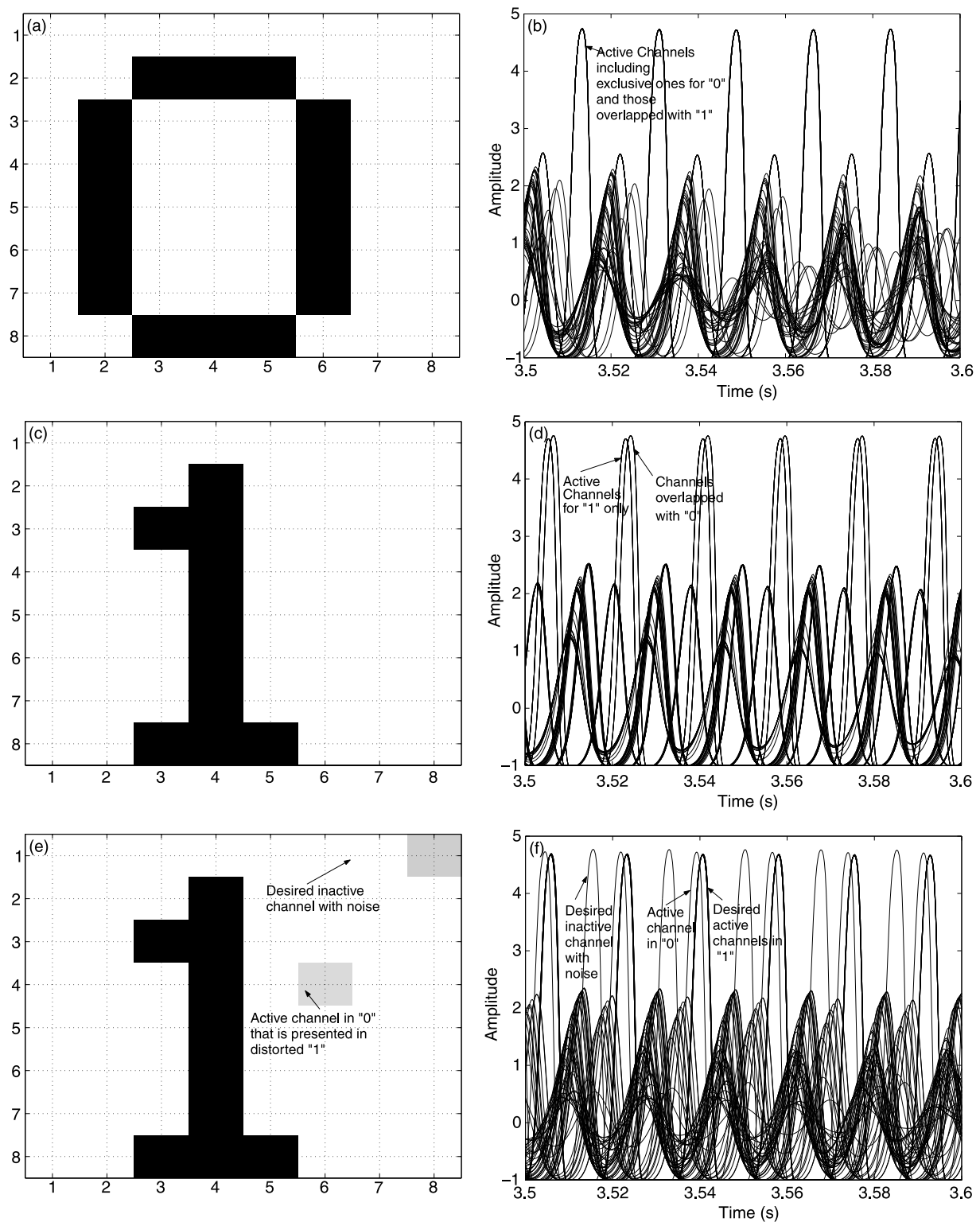

Figure 5. RKII network as an associative memory. The individual RKII set is configured with $K_{m g}=1, K_{g m}=-6$, and positive input $P=3$. For two linearly coupled RKII sets, Case 1 couplings are set as $K_{m m}=0.2$ and $K_{g g}=-0.4$. Case 2 couplings are $K_{m m}=0.2$ and $K_{g g}=-0.1$. Case 3 couplings are $K_{m m}=0.1$ and $K_{g g}=-0.8$. Case 4 couplings are $K_{m m}=0.2$ and $K_{g g}=$ -0.3 . All couplings are further normalized according to the number of channels in the network. 0.8 is set to be the threshold of correlation coefficients between two channels. Two channels with a correlation value larger than the threshold are assigned to the same group. (a) Stored pattern "0." (b) Network response to input pattern "0." Desired active channels are almost identically synchronized (small phase differences exist between the active channels for " 0 " and those overlapped with "1") and have a larger amplitude of oscillation. Desired inactive channels have 


\section{CONCLUSIONS}

Synchronized oscillations are believed to play a key role in many biological models, especially for the purpose of information processing. Based on our previous work on analyzing how to control an RKII network to achieve desired behaviors, we proposed to use synchronization as a computational primitive in an RKII network. Information regarding collective behaviors in such networks is used to design the readout. Network parameters (coupling strengths among different RKII sets) are designed specifically to achieve desired responses. Two applications are presented. By utilizing two coupled RKII sets as a logic computation unit, the intrinsic structure of the coupled sets provides us with very flexible configurations to implement any logic gates, and the system can realize universal sets of logic operations. The potential capacity of this network is very large; however, the controllability of all the attractors in the output space is not guaranteed and needs to be further investigated.

In the second application, we demonstrated how to design an RKII network as an associative memory by considering synchronization of groups of oscillators in the output space. Finding parameter values in this case is straightforward compared to conventional learning algorithms. Synchronization as a readout also has advantages over energy-based methods because correlation of states is the way the network represents input patterns in the output space. Especially, the synchrony readout performs much better in the presence of noisy patterns when energy-based methods may fail. There are still many problems that need to be explored. Capacity and robustness of the network in terms of stored patterns and signal-to-noise ratio need to be investigated. In the applications pursued so far, only fixed point solutions and synchronized oscillations have been used for encoding information. This strategy may severely limit the capacity of neural-network-based models. It would be very interesting to develop schemes where desynchronized oscillations,

\section{Figure 5 caption continued:}

a small amplitude of oscillation and are desynchronized with active channels. Using the criterion given in Section 4.2, pattern "0" can easily be recovered to the stored pattern as shown in (a). (c) Stored pattern "1." (d) Network response to input pattern "1." Phase differences among desired active channels are slightly larger than those presented in the case of pattern " 0 ." However, using the given threshold, pattern "1" can be perfectly recovered. (e) Colored input pattern "1." Positive inputs $(P=3)$ as noise are fed into a desired inactive channel and a active channel for " 0 " only. Image shows the total amount of input received in each synchronized group $\left(\mathbf{O}_{i}=\right.$ $\left.\sum_{k \in G_{i}} A_{k}\right)$. Although noise increases the received input values for undesired channels, those channels tend to be grouped separately, so the readout value will be kept low. When more channels are distorted by noise, as long as they are desynchronized, the final readout will always be much lower than the value of large number of active channels that are grouped together. (f) In the time domain, we see that undesired channels interfere with desired active channels. In this case, simple amplitude- or energy-based readout will fail the test. However, correlation between those colored channels and desired channels will still divide them into two different groups. Pattern "1" can still be perfectly recovered by choosing the group of channels that have maximum readout in (e). 
quasiperiodic oscillations, and possibly chaos, together with fixed point solutions and synchronized oscillations, can all be actively used to encode information.

\section{Acknowledgments}

This work was partially supported by ONR N00014-1-1-0405.

\section{References}

1. Freeman WJ. Mass action in the nervous system. New York: Academic; 1975.

2. Hopfield J. Neural networks and physical systems with emergent collective computational abilities. Proc Natl Acad Sci 1982;79:2554-2558.

3. Chua L. Cnn: A paradigm for complexity. New York: World Scientific Publishing Co.; 1998.

4. Chua L, Yang L. Cellular neural networks: Theory. IEEE Trans Circ Syst 1988;35: $1257-1272$.

5. Wang D. Relaxation oscillators and networks. In: Webster JG, editor. Wiley encyclopedia of electrical and electronics engineering. New York: Wiley; 1999.

6. Chen K, Wang D. A dynamically coupled neural oscillator network for image segmentation. Neural Netw 2002;15:423-439.

7. Grossberg S. Pattern learning by functional-differential neural networks with arbitrary path weights. In: Schmitt K, editor. Delay and functional differential equations and their applications. New York: Academic; 1972. pp 121-160.

8. Llinas R. I of the vortex: From neurons to self. Cambridge, MA: MIT Press; 2001.

9. Hopfield J. Neurons with graded response have collective computational properties like those of two-state neurons. Proc Natl Acad Sci 1984;81:3088-3092.

10. Kaplan D, Glass L. Understanding nonlinear dynamics. New York: Springer-Verlag; 1995.

11. Kelso S. Dynamic patterns: The self-organization of brain and behavior. Cambridge, MA: MIT Press; 1995.

12. Yao Y, Freeman W, Burke B, Yang Q. Pattern recognition by a distributed neural network: An industrial application neural networks. Neural Netw 1991;4:103-121.

13. Kozma R, Freeman W. Chaotic resonance-methods and applications for robust classification of noisy and variable patterns. Int J Bifurcat Chaos Appl Sci Eng 2001;11:1607-1629.

14. Principe J, Tavares V, Harris J, Freeman W. Design and implementation of a biologically realistic olfactory cortex in analog VLSI. Proc IEEE 2001;89:569-571.

15. Tavares V. Design and implementation of a biologically realistic olfactory cortex model. Ph.D. thesis. Gainesville, FL: University of Florida; 2001.

16. Nekorkin VI, Velarde MG. Synergetic phenomena in active lattices. New York: SpringerVerlag; 2002.

17. De Feo O. Tuning chaos synchronization and anti-synchronization for applications in temporal pattern recognition. Int J Bifur Chaos Appl Sci Eng 2005;12:3905-3921.

18. Henkel R. Synchronization, coherence-detection and three-dimensional vision; 2000. Available at: http://citeseer.ist.psu.edu/henkel00synchronization.html.

19. Gutierrez-Galvez A, Gutierrez-Osuna R. Pattern completion through phase coding in population neurodynamics. Neural Netw 2003;16:649-656.

20. Han SK, Kurrer C, Kuramoto Y. Dephasing and bursting in coupled neural oscillators. Phys Rev Lett 1995;75:3190-3193.

21. Bressloff PC, Coombes S. Desynchronization, mode locking, and bursting in strongly coupled integrate-and-fire oscillators. Phys Rev Lett 1998;81:2168-2171.

22. Pastordiaz I, Lopezfraguas A. Dynamics of two coupled van der Pol oscillators. Phys Rev E 1995;52:1480-1489.

23. Bressloff PC, Coombes S. Synchrony in an array of integrate-and-fire neurons with dendritic structure. Phys Rev Lett 1997;78:4665-4668. 
24. Reddy DVR, Sen A, Johnston GL. Time delay induced death in coupled limit cycle oscillators. Phys Rev Lett 1998;80:5109-5112.

25. Herrero R, Figueras M, Rius J, Pi F, Orriols G. Experimental observation of the amplitude death effect in two coupled nonlinear oscillators. Phys Rev Lett 2000;84:5312-5315.

26. Reddy DVR, Sen A, Johnston GL. Experimental evidence of time-delay-induced death in coupled limit-cycle oscillators. Phys Rev Lett 85;2000:3381-3384.

27. Singer W, Gray CM. Visual feature integration and the temporal correlation hypothesis. Annu Rev Neurosci 1995;18:555-586.

28. Phillips WA, Singer W. In search of common foundations of cortical computations. Behav Brain Sci 1997;20:657.

29. Keil A, Mueller MM, Ray WJ, Gruber T, Elbert T. Human gamma band activity and perception of a gestalt. J Neurosci 1999;19:7152-7161.

30. Freeman WJ. Characteristics of the synchronization of brain activity imposed by finite conduction velocities of axon. Int J Bifurcat Chaos Appl Sci Eng 2000;10:2307-2322.

31. Xu D, Principe J. Dynamical analysis of neural oscillators in an olfactory cortex model. IEEE Trans Neural Netw 2004;15:1053-1062.

32. Xu D, Gao J, Principe J. Synchronization of two coupled oscillators in Freeman's olfactory cortex model. Phys Lett A 2006 (submitted).

33. Xu D, Principe J, Harris J. Logic computation using coupled neural oscillators. IEEE Int Symp Circ Syst 2004;5:788-791.

34. Kocarev L, Parlitz U. Generalized synchronization, predictability, and equivalence of unidirectionally coupled dynamical systems. Phys Rev Lett 1996;76:1816-1819.

35. Brown R, Rulkov NF. Designing a coupling that guarantees synchronization between identical chaotic systems. Phys Rev Lett 1997;78:4189-4192.

36. Haykin S. Neural networks: A comprehensive foundation. Englewood Cliffs, NJ: Prentice Hall; 1998.

37. Principe JC, Euliano NR, Lefebvre WC. Neural and adaptive systems: Fundamentals through simulation. New York: Wiley; 1999. 